\title{
Some numerical methods of thermal dosimetry for applications in bioelectromagnetics
}

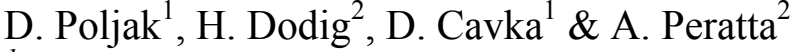 \\ ${ }^{1}$ University of Split, Croatia \\ ${ }^{2}$ Wessex Institute of Technology, UK
}

\begin{abstract}
Some thermal dosimetry methods for the analysis of human exposure to high frequency (HF) electromagnetic radiation are reviewed in this work. The formulation is based on the bio-heat transfer equation. Calculating the distribution of specific absorption rate (SAR) inside the biological body provides an assessment of a related thermal response to HF electromagnetic fields. The temperature increase is obtained by solving the bio-heat transfer equation for different exposure scenarios via Boundary Element Method (BEM) and Finite Element Method (FEM), respectively. Some illustrative computational examples are presented in the paper.
\end{abstract}

\section{Introduction}

The dominant and well-established biological effect due to human exposure to high frequency (HF) electromagnetic radiation is tissue heating [1-6]. Thermal effects are often defined in terms of energy deposition higher than the thermoregulatory capacity of the human body [3]. Consequently, adverse effects can occur when the total power absorbed by the body is large enough to cause protective mechanisms for heat control to break down, thus resulting in uncontrolled rise in the body temperature (hyperthermia).

The hazardous field levels can be quantified analysing the thermal response of the human body exposed to HF radiation. The problem to be considered is by itself twofold. First, the rate of power deposition in tissue due to HF radiation has to be calculated; and second the related temperature distribution within the body has to be assessed. The electromagnetic analysis itself includes the incident 
field dosimetry (modeling of the radiation sources) and internal field dosimetry (modeling of the field induced in the body).

This paper reviews the previous work by the authors related to models and methods of thermal dosimetry [5-9].

Thermal behaviour of the human body is analysed through the solution of the bio-heat transfer equation representing the balance between internal heat generation due to metabolism, internal convective heat transfer due to blood flow, external interaction by convection and radiation and cooling of the skin by sweating and evaporation and finally absorbed energy due to radiation [4].

In this paper, the bio-heat transfer equation is numerically solved using the Boundary Element Method (BEM) and the Finite Element Method (FEM) for different models of the human body. The thermal response of both simplified and realistic body model, respectively, is obtained. Also, a temperature increase in the human eye model is determined.

\section{Formulation}

The rate of volumetric heat generation due to the electromagnetic radiation is obtained from HF electromagnetic dosimetry methods [6-9].

The key point in of internal dosimetry pertaining to HF bioelectromagnetics is related to the level and distribution of the electromagnetic energy absorbed by the human body This effect is standardly quantified in terms of the Specific Absorption Rate (SAR) being defined as the mass averaged power $P$, i.e. rate of energy $W$ absorbed by the biological body:

$$
S A R=\frac{d P}{d m}=\frac{d}{d m} \frac{d W}{d t}=C \frac{d T}{d t}
$$

where $C$ is the specific heat capacity of actual tissue, $T$ is the temperature while $t$ denotes time.

On the other hand, the localized SAR directly depends on the internal field, as well, and, therefore, the main task of the internal dosimetry is the assessment of the electric field distribution inside the human body.

Thus, $S A R$ in tissue is proportional to the square of the internal electric field:

$$
S A R=\frac{d P}{d m}=\frac{d P}{\rho d V}=\frac{\sigma}{2 \rho}|E|^{2}
$$

where $E$ is the peak value of the electric field, $\sigma$ is the conductivity of the tissue and $\rho$ is the tissue density.

Generally, SAR values are strongly dependent on the incident field parameters, the electrical properties of the exposed body, reflecting and absorbing effects of the nearby objects, etc. When the external field is parallel to the human body the whole body SAR reaches maximal values. 
The thermal steady state phenomena in the body are analyzed using the stationary bio-heat transfer equation [4]:

$$
\nabla \cdot(\lambda \nabla T)+W_{b} C_{p b}\left(T_{a}-T\right)+Q_{m}+Q_{E M}=0
$$

where: $\lambda$ is the thermal conductivity, $W_{b}$ is the volumetric perfusion rate, $T$ is the tissue temperature, $C_{p b}$ is the specific heat of blood, $T_{a}$ is the arterial temperature, $Q_{m}$ is the power produced by metabolic process and $Q_{E M}$ is the electromagnetic power deposition - a source term directly related to $S A R$ and deduced from electromagnetic analysis:

$$
Q_{E M}=\frac{\sigma}{2}|E|^{2}=\rho \cdot S A R
$$

Furthermore, the boundary condition at the interface between skin and air, and is given by:

$$
q=H\left(T_{s}-T_{a}\right)
$$

where $q$ is the heat flux density defined as:

$$
q=-\lambda \frac{\partial T}{\partial n}
$$

while $H, T_{s}$ and $T_{a}$ denote, respectively, the convection coefficient, the temperature of the skin, and the temperature of the air.

\section{Models and methods}

Various models for different exposure scenarios are discussed in the paper; simplified cylindrical body model, three-dimensional anatomically based body model and two-dimensional model of the human eye. Certain variants of BEM and FEM have been implemented to solve related problems.

For the sake of completeness, these numerical procedures are outlined in Appendix A and B, respectively.

\section{Computational examples}

First example deals with a simplified cylindrical body model exposed to radiation of a base station antenna system mounted on a roof-top of a $35 \mathrm{~m}$ high building. The maximal value of the radiated electric field, tangential to the body, at $30 \mathrm{~m}$ distance of the antenna system main beam in a nearby flat calculated via ray-tracing algorithm [8] is $15 \mathrm{~V} / \mathrm{m}$. The calculation of SAR distribution within a cylindrical body representation, Fig 1, is available in [8], as well.

Temperature distribution inside the body due to the absorbed electromagnetic energy is determined by solving the bio-heat transfer equation (3) by means of the dual reciprocity boundary element method (DR-BEM) $[8,10]$. The average thermal properties of the human body model are as follows $[5,6]$ : 
$\lambda=0.545 \mathrm{~W} / \mathrm{m}^{\circ} \mathrm{C}, W_{b}=0.433 \mathrm{~kg} / \mathrm{m}^{3} \mathrm{~s}, Q_{m}=703.5 \mathrm{~W} / \mathrm{m}^{3}$ and $C_{p b}=3475 \mathrm{~J} / \mathrm{kg}^{\circ} \mathrm{C}$, assuming the homogenous body is composed of muscular tissue. The arterial blood temperature and tissue density is assumed to be $T_{a}=36.7^{\circ} \mathrm{C}$ and $\rho=1000 \mathrm{~kg} / \mathrm{m}^{3}$, respectively. The obtained temperature distribution with related heat flux field is shown in Fig 2.

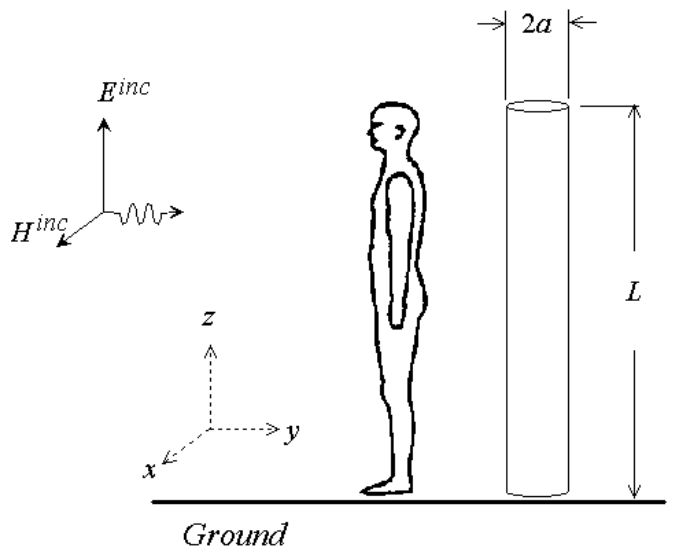

Figure 1: The cylindrical antenna model of the human body.

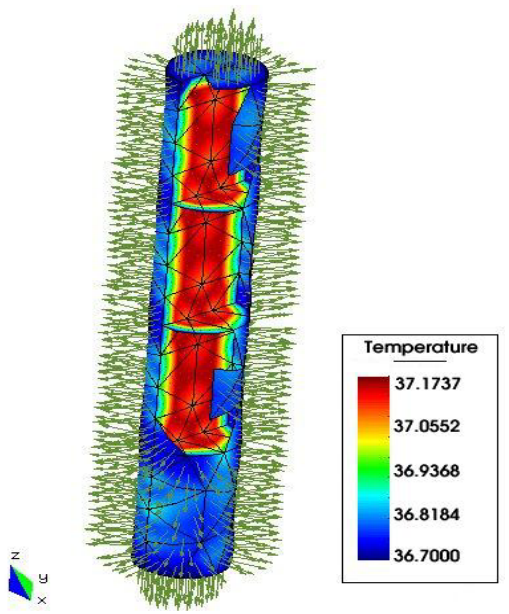

Figure 2: Temperature distribution in the cylindrical body model and normal heat flux [8].

The total temperature increase obtained for the cylindrical body model inside the muscle tissue, due to the both metabolic heat production and electromagnetic power deposition is around $37.17^{\circ} \mathrm{C}$. 
The maximum calculated temperature rise is $\Delta T=4.66^{*} 10^{-6 \circ} \mathrm{C}$ and found to be rather negligible.

Next example deals with the realistic, anatomically based, model of the human body, Fig 3a, with average thermal properties (muscle tissue) as in the previous example. The finite element mesh of the homogeneous body exposed to GSM base station radiation is shown in Fig $3 \mathrm{~b}$. The corresponding value of external field is taken to be $0.16 \mathrm{~V} / \mathrm{m}$ [9].

Figure 4 shows the temperature distribution inside the body.

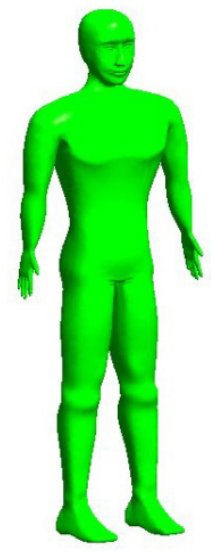

(a)

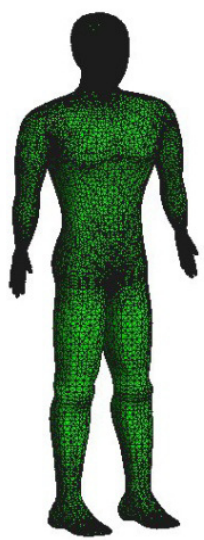

(b)

Figure 3: (a) Human body model. (b) FEM mesh.

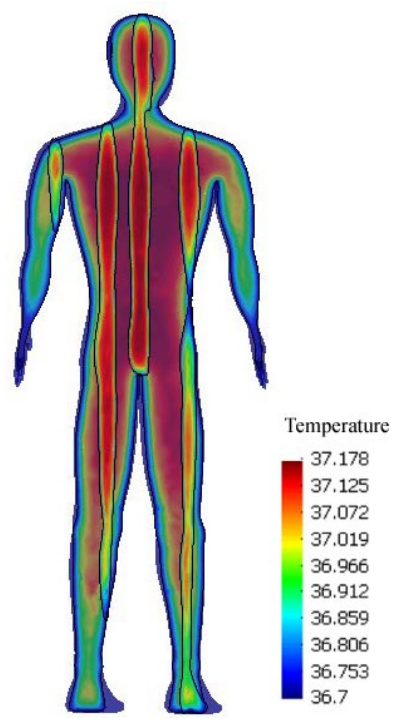

Figure 4: Temperature distribution inside the body for $\mathrm{Q}_{\mathrm{SAR}}=0$ [9]. 
The maximal temperature computed from the finite element model of the body with muscle temperature properties, due to both metabolic heat production and electromagnetic power deposition is around $37.178^{\circ} \mathrm{C}$. Consequently, the maximal temperature increase generated by power deposition due to GSM base station radiation is $\Delta T=1.75 * 10^{-9 \circ} \mathrm{C}$ which is rather negligibly small.

The last example is related to two-dimensional model of the human eye exposed to the incident transverse magnetic (TM) plane wave with power density $5 \mathrm{mWcm}^{-2}$ at frequency $0.85 \mathrm{GHz}$.

Figure 6 shows the temperature increase in the human eye due to microwave radiation at $0.85 \mathrm{GHz}$. The corresponding logarithmic plot of average and maximum temperature increase in the human eye in the frequency range from 0.7 to $4.4 \mathrm{GHz}$ is shown in Fig 7 .

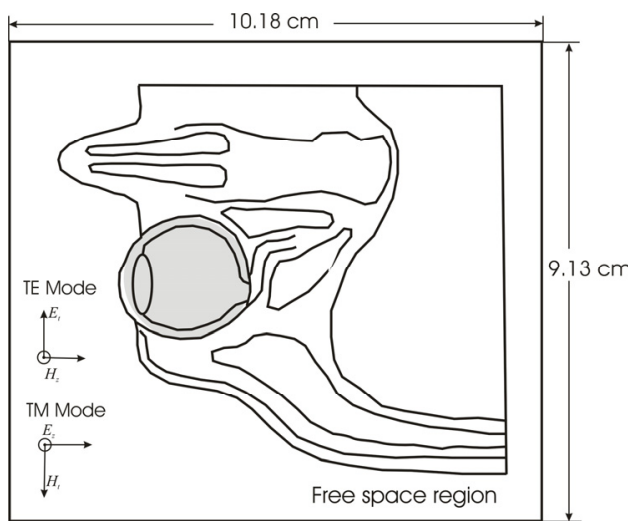

(a)

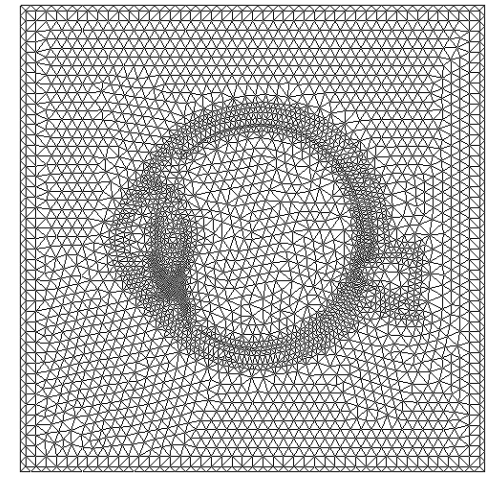

(b)

Figure 5: (a) A top view to the calculation domain. (b) FEM mesh for the eye.

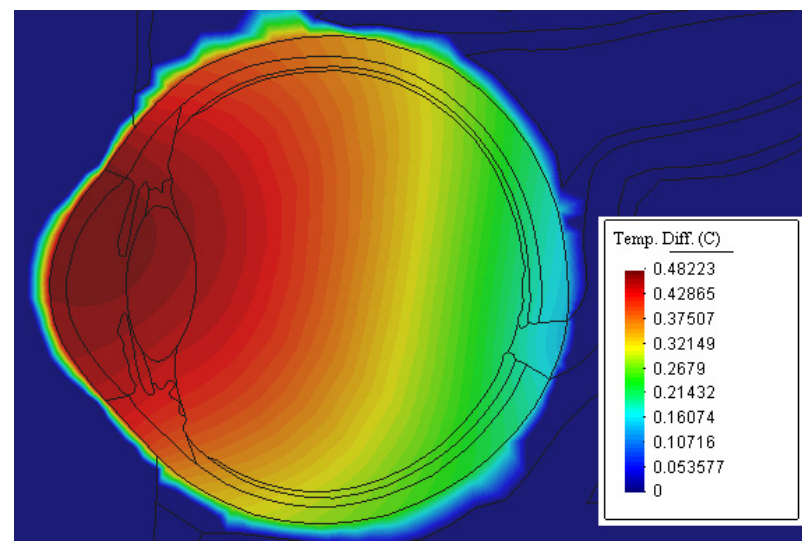

Figure 6: Temperature rise in the human eye due to the TM plane wave with frequency $\mathrm{f}=0.85 \mathrm{GHz}$ and power density $5 \mathrm{mWcm}^{-2}[7]$. 


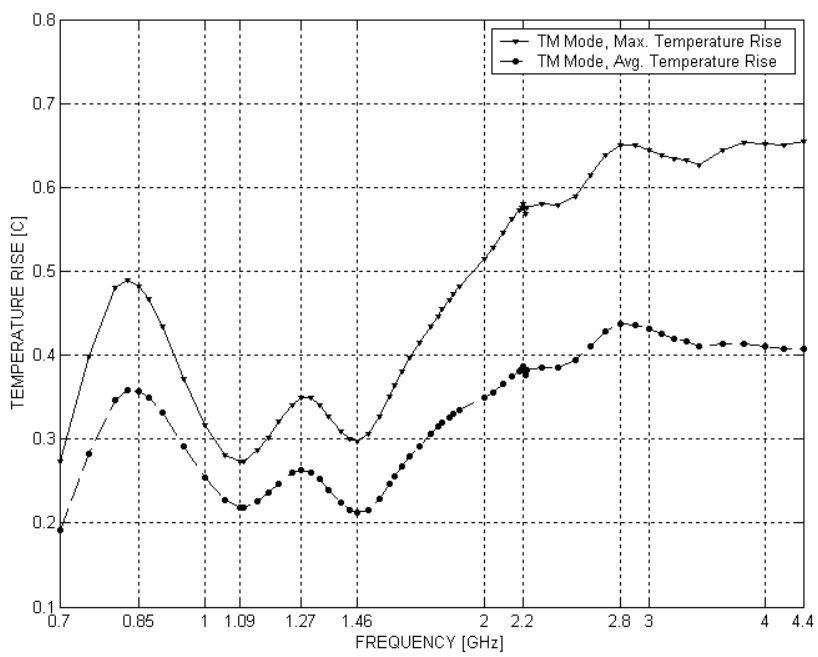

Figure 7: Logarithmic plot of average and maximum temperature rise in the human eyes due to TM plane wave in the frequency range: 0.7$4.4 \mathrm{GHz}$, and power density $5 \mathrm{mWcm}^{-2}$.

Results shown in Fig 7 reveal a peak temperature increase in the eye near the GSM frequency of $900 \mathrm{MHz}$.

\section{Conclusion}

The paper deals with some thermal dosimetry methods for the study of human exposure to high frequency (HF) electromagnetic fields. The formulation is based on the stationary bio-heat transfer equation. Calculating the distribution of specific absorption rate (SAR) inside the biological body it is possible to determine a related thermal response to $\mathrm{HF}$ electromagnetic fields. The temperature increase is then assessed by solving the bio-heat transfer equation for different exposure scenarios via the Boundary Element Method (BEM) and the Finite Element Method (FEM), respectively. Some illustrative computational examples are presented throughout the paper.

\section{Appendix A: boundary element procedures}

The stationary bio-heat transfer equation for the simplified cylindrical body model is solved via Dual Reciprocity Boundary Element Method (DR-BEM). The governing equation used for steady bio-heat transfer is stationary Pennes equation (3) can be written in the following form:

$$
\nabla(\lambda \cdot \nabla T)=f(T)
$$


where:

$$
f(T)=W_{b} \cdot C_{p b}\left(T-T_{a}\right)-Q_{m}-Q_{E M}
$$

Applying the Green integral representation a following integral relation for an isolated subdomain can be written:

$$
c(\xi) \phi(\xi)+\int_{\Gamma_{s}} \phi \frac{\partial \phi^{*}}{\partial n} d \Gamma-\int_{\Gamma_{s}} \frac{\partial \phi}{\partial n} \phi^{*} d \Gamma=\int_{\Omega_{s}} \phi^{*} \rho d \Omega_{s}
$$

where $\phi=T-T_{a}$ and

$$
\phi^{*}=\frac{1}{4 \pi r}
$$

is the corresponding three-dimensional fundamental solution.

Discretization of expression (A4) with $N_{e}$ elements leads to the following expression:

$$
c_{i} \phi_{i}+\sum_{j=1}^{N_{e}} \int_{\Gamma_{s, j}} \phi \frac{\partial \phi^{*}}{\partial n} d \Gamma-\sum_{j=1}^{N_{e}} \int_{\Gamma_{s, j}} \frac{\partial \phi}{\partial n} \phi^{*} d \Gamma=\int_{\Omega_{s}} \rho \phi^{*} d \Omega_{s}
$$

where $i$ stands for the source point and $\Gamma_{s, j}$, represents the $j$-th boundary element of $\Omega_{s}$.

The present implementation is based on the isoparametric approach with quadratic interpolation functions over triangular and quadrilateral elements surrounding a region or subdomain of any arbitrary shape.

Using quadratic elements, the potential or its normal derivative, at any point of the $j$-th boundary element can be written in terms of their corresponding values at the $n_{k}$ collocation nodes by means of the interpolation functions $\psi_{a}$.

$$
\begin{gathered}
\phi(\xi)=\sum_{a=1}^{n_{k}} \psi_{a}(\xi) \phi_{a} \\
\frac{\partial \phi(\xi)}{\partial n}=\sum_{a=1}^{n_{k}} \psi_{a}(\xi) \phi_{a}
\end{gathered}
$$

The dimensionless coordinate $\xi$ spans from the computational domain to the physical patch or boundary element.

Performing certain mathematical manipulations and by applying Green's identity to equation (A5) one obtains corresponding matrix equation whose solution yields the values of the temperature increase. The DR-BEM is described in detail elsewhere, e.g. in $[8,10]$. 


\section{Appendix B: finite element procedures}

The stationary bio-heat transfer equation for three-dimensional model of the body and two-dimensional eye model is handled via FEM.

The bio heat transfer equation (3) can be written in the form of the inhomogeneous Helmholtz-type equation:

$$
\nabla(\lambda \nabla T)-W_{b} C_{p b} T=-\left(W_{b} C_{p b} T_{a}+Q_{m}+Q_{E M}\right)
$$

with an associated Neumann boundary condition defined with (5) and (6).

The standard finite element discretization of Helmholtz equation results in the following matrix equation:

$$
[K]\{T\}=\{M\}+\{P\}
$$

where $[\mathrm{K}]$ is the finite element matrix given by:

$$
K_{j i}=\int_{\Omega_{e}} \nabla f_{j}\left(\lambda \nabla f_{i}\right) d \Omega_{e}+\int_{\Omega_{e}} W_{b} C_{p b} f_{j} f_{i} d \Omega_{e}
$$

while $\{M\}$ denotes the flux vector:

$$
M_{j}=\int_{\Gamma_{e}} \lambda \frac{\partial T}{\partial n} f_{j} d \Omega_{e}
$$

and $\{P\}$ stands for the source vector:

$$
p_{j}=\int_{\Omega_{e}}\left(W_{b} C_{p b} T_{a}+Q_{m}+Q_{E M}\right) f_{j} d \Omega_{e}
$$

In this paper the calculation is carried out using the linear base functions and triangular elements.

\section{References}

[1] R.W.Y. Habash: Electromagnetic Fields and Radiation, Marcel Dekker, New York, 2002.

[2] E.R. Adair, R.C. Petersen: Biological Effects of Radiofrequency/Microwave Radiation, IEEE Trans. Microwave Theory and Tech., Vol. 50, No 3, pp. 953-962, March 2003.

[3] International Commission on Non-Ionizing radiation Protection (ICNIRP): Guidelines for Limiting Exposure to Time-Varying, Electric, Magnetic and Electromagnetic Fields (up to 300GHz), Health Phys., Vol. 74, 4 (1998), 494-522.

[4] A. Hirrata, T.Shizoava: Correlation of Maximum Temperature Increase and Peak SAR in the Human Head Due to Handset Antennas, IEEE Trans. Microwave Theory and Tech., Vol. 51, No 7, pp. 1834-1841, July 2003. 
[5] D.Poljak, Elsevier 2011. Poljak D (2011) Electromagnetic fields: Environmental exposure. In: Nriagu JO (ed.) Encyclopedia of Environmental Health, volume 2, pp. 259-268 Burlington: Elsevier.

[6] D.Poljak: Human Exposure to Electromagnetic Fields, WIT Pres, Southampton-Boston, 2003.

[7] H.Dodig, A.Peratta, D.Poljak: Analysis Method for the Heating of the Human Eye Exposed to High Frequency Electromagnetic Fields, Journal of Communications Software and Systems, Vol. 3, No 1, March 2007., pp. 3 - 10.

[8] Dragan Poljak, Andres Peratta, Carlos A. Brebbia: "The boundary element electromagnetic-thermal analysis of human body exposure to base station antennas radiation", Engineering Analysis with Boundary Elements 28 (2004) 763-770.

[9] D.Čavka, Finite Element Thermal Model of the Human Exposed to Electric Field Generated from GSM Base Station, SoftCOM 2006.

[10] P.W.Partridge, C.A. Brebbia, L.C. Wrobel " The Dual Reciprocity Boundary Element Method", Computational Mechanics Publications, Southampton, Boston, 1992. 